\title{
A general few-projection method for tomographic reconstruction of samples consisting of several distinct materials
}

\author{
Glenn R. Myers, ${ }^{1, a)}$ C. David L. Thomas, ${ }^{2}$ David M. Paganin, ${ }^{3,4}$ Timur E. Gureyev, ${ }^{4}$ and \\ John G. Clement ${ }^{2}$ \\ ${ }^{1}$ Department of Applied Mathematics, The Australian National University, Canberra ACT 0200, Australia \\ ${ }^{2}$ Melbourne Dental School, The University of Melbourne, Melbourne 3010, Australia \\ ${ }^{3}$ School of Physics, Monash University, Clayton 3800, Australia \\ ${ }^{4}$ CSIRO Materials Science and Engineering, PB 33, Clayton South, 3169 Australia
}

(Received 24 September 2009; accepted 5 December 2009; published online 12 January 2010)

\begin{abstract}
We present a method for tomographic reconstruction of objects containing several distinct materials, which is capable of accurately reconstructing a sample from vastly fewer angular projections than required by conventional algorithms. The algorithm is more general than many previous discrete tomography methods, as: (i) a priori knowledge of the exact number of materials is not required; (ii) the linear attenuation coefficient of each constituent material may assume a small range of a priori unknown values. We present reconstructions from an experimental x-ray computed tomography scan of cortical bone acquired at the SPring-8 synchrotron. () 2010 American Institute of Physics. [doi:10.1063/1.3279150]
\end{abstract}

Since its inception, computed tomography (CT) has become a method of choice for nondestructive threedimensional (3D) imaging in fields such as medicine, biology, and materials science. ${ }^{1}$ The number of angular projections required for an unambiguous reconstruction $\left(p_{\mathrm{ct}}\right)$ is proportional to the desired spatial resolution. ${ }^{1}$ Consequently, conventional methods require hundreds or thousands of images to reconstruct the object at a useful resolution.

Though the work in this letter generalizes easily to more complex imaging geometries, we assume an imaging setup in which an object of interest is illuminated by penetrating plane-parallel radiation (e.g., X-rays, neutrons, electrons, etc.). A two-dimensional (2D) position-sensitive detector is then placed on the opposite side of the object, normal to the direction of radiation propagation. The object is gradually rotated through $180^{\circ}$, and a sequence of images is taken at a number of different viewing (projection) angles. ${ }^{1}$ Assuming refraction within the object is negligible and the linear attenuation coefficient $[\mu(\mathbf{x})]$ of the object is much less than the wave-number of the incident radiation, the intensity map recorded at the detector will depend directly on $\mu(\mathbf{x})$, integrated along lines normal to the detector. ${ }^{2}$

The proportional relation between spatial resolution (number of pixels) in the reconstruction and $p_{\mathrm{ct}}$ assumes nothing about the object, except that it fits entirely in the field of view of the detector. In discrete tomography (DT) it is assumed that the object is composed of a known number of nonoverlapping materials, each of a constant density. Consequently, for an $\mathrm{N}$-material object obeying the assumptions of DT, $\mu(\mathbf{x})$ may assume only $N$ discrete values. ${ }^{3-11}$ The number of projections actually collected $(p)$ can thus be well below $p_{\mathrm{ct}}$. A recent algorithm allows reconstruction of singlematerial objects which display small variations in density. ${ }^{12}$ The majority of DT reconstruction algorithms also assume that the $\mu(\mathbf{x})$ of each material are known a priori. ${ }^{3-11}$

Unfortunately, many objects of interest violate DT assumptions. For example, bone density in biological speci-

${ }^{\text {a)} E l e c t r o n i c ~ m a i l: ~ m y e r s . g l e n n . r @ g m a i l . c o m . ~}$ mens is rarely constant across the sample, and is often a quantity of interest (i.e., unknown a priori). ${ }^{13}$ This highlights the need for a CT reconstruction algorithm which makes comparatively weak a priori assumptions, and is applicable to a wide range of few-material objects which fall outside the domain of validity of existing DT algorithms. In particular, we target fields which will benefit from fast, high-throughput imaging, as well as imaging of samples which are too fragile to survive the dose delivered during a conventional CT scan.

We consider the case of an object composed of a small (though unknown) number of unknown, distinct materials. Each of these materials may display small fluctuations in density [and thus $\mu(\mathbf{x})$ ] about an unknown mean. We now introduce the object "histogram"; the number of voxels $h(m)$ in the histogram bin with index $m \in[0,1, \ldots, M]$ is defined as the number of voxels for which $m w<\mu(\mathbf{x}) \leq(m+1) w$, where $w=\{\max [\mu(\mathbf{x})]-\min [\mu(\mathbf{x})]\} / M$ is the bin width. If we plot the histogram, the graph would show several distinct peaks, each corresponding to a different material. The exact location and number of these peaks is unknown.

We present an iterative reconstruction algorithm which enforces a strongly peaked structure in the histogram of the reconstruction while maintaining consistency with the recorded data, henceforth referred to as a histogram-based CT (HB-CT) reconstruction algorithm. The behavior of the HB-CT algorithm is investigated for both; (i) simulated one-, two-, and three-material phantoms and (ii) reconstruction of cortical bone microstructure from experimental data collected at the SPring-8 BL20B2 X-ray synchrotron beamline. In the latter case we achieve $p=p_{\mathrm{ct}} / 10$, leading to a $10 \times$ reduction in scanning time and absorbed dose.

We are concerned with recovering the $3 \mathrm{D}$ distribution of $\mu(\mathbf{x})$ in a nonrefracting object for which $\mu(\mathbf{x}) \ll 2 \pi / \lambda$, implying that the object is sufficiently weakly scattering for the projection approximation to be applicable. ${ }^{2} \lambda$ denotes the wavelength of the illumination. We assume the standard plane-parallel CT imaging geometry discussed above, in which a set of projection images: ${ }^{1,2}$ 


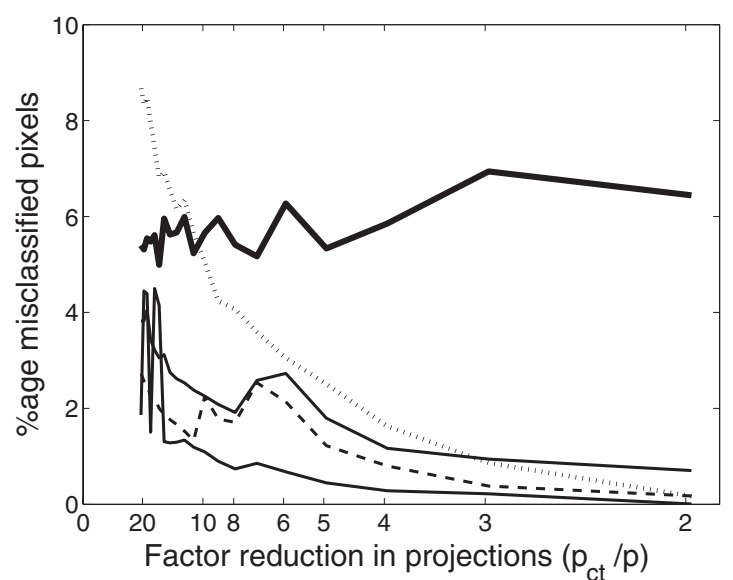

FIG. 1. Error in HB-CT reconstructions through a one (thin solid line), two (0\% noise-dashed line, $2 \%$ noise-dashed and dotted, 5\% noise- thick solid line), and three (dotted line) material phantom.

$$
g(\mathbf{r}, \theta)=(\mathcal{P} \mu)(\mathbf{r}, \theta)
$$

$$
\equiv \int_{-\infty}^{\infty} \mu\left(t \cos \theta-r_{1} \sin \theta, r_{1} \cos \theta+t \sin \theta, r_{2}\right) \mathrm{d} t,
$$

is collected at several different projection angles $\theta \in[0, \pi)$ about the object. We use $\mathbf{x}=\left(x_{1}, x_{2}, x_{3}\right)$ to denote a rotating Cartesian coordinate frame fixed to the object and oriented such that $x_{3}$ points upwards along the rotation axis, $\mathbf{r}$ $=\left(r_{1}, r_{2}\right)$ to denote Cartesian coordinates in the detector plane, and introduce the projection operator $\mathcal{P}$. In the case of illumination by monochromatic x-rays of incident intensity $I_{\text {in }}, g(\mathbf{r}, \theta)$ may be recovered directly from the recorded intensity $I(\mathbf{r}, \theta)=I_{\text {in }} \exp [-g(\mathbf{r}, \theta)]$ in the contact plane.

Throughout this letter we will make certain assumptions allowing us to retrieve $\mu(\mathbf{x})$ from far fewer than the $p_{\mathrm{ct}}$ $=\pi q / 2$ angular projections usually required for a reconstruction with $q \times q \times q$ voxels. ${ }^{1,3}$ Specifically, we assume that (i) our object is composed of a small number of materials; and (ii) the ranges of $\mu(\mathbf{x})$ corresponding to each material are distinct. At no stage do we assume knowledge of the exact number of materials within an object, or the linear attenuation coefficient of any given material. Furthermore, we do not assume a single value of refractive index per material. Our reconstruction algorithm addresses the more general case in which each material corresponds to a distinct range of refractive indices due to, e.g., small spatial fluctuations in density.

We begin by using filtered backprojection (FBP) to make an initial estimate: ${ }^{1}$

$$
\begin{aligned}
\mu^{0}(\mathbf{x})= & (\mathcal{B} g)(\mathbf{x}) \equiv \int_{0}^{\pi} \int_{-\infty}^{\infty}\left|\xi_{1}\right|(\mathcal{F} g)\left(\xi_{1}, x_{3}, \theta\right) \\
& \times \exp \left[2 \pi i \xi_{1}\left(x_{2} \cos \theta-x_{1} \sin \theta\right)\right] \mathrm{d} \xi_{1} \mathrm{~d} \theta
\end{aligned}
$$

where $\mathcal{B}$ is the FBP operator and $\mathcal{F}$ denotes the one dimensional Fourier transform with $\xi_{1}$ dual to $r_{1}$. We then recursively refine the estimated linear attenuation coefficient according to

$$
\mu^{(n+1)}(\mathbf{x})=\mu^{(n)}(\mathbf{x})+\gamma^{(n)} \mathcal{B}\left[g(\mathbf{r}, \theta)-\left(\mathcal{P H} \mu^{(n)}\right)(\mathbf{r}, \theta)\right],
$$

where $\gamma^{(n)}$ is a constant, and the superscript $(n)$ denotes the $n$th iteration of the recursive scheme. Equation (3) forms our $\mathrm{HB}-\mathrm{CT}$ reconstruction algorithm.

$\mathcal{H}$ is an operator that promotes peaks in the histogram of the object, and thus consistency with our a priori information. In this letter, $\mathcal{H}$ is defined as follows:

1. Determine and store "top-down" ordering of voxels from $\mu^{(n)}(\mathbf{x})$, where $\mu^{(n)}(\mathbf{x})$ is highest, second highest, etc.

2. Calculate histogram $h^{(n)}(m)$ of $\mu^{(n)}(\mathbf{x})$.

3. Soft-threshold to obtain new histogram $h^{(n)}(m)=h^{(n)}(m)$ $-\tau, \quad \forall m: h^{(n)}(m)>\tau$, and $h^{(n)}(m)=0, \quad \forall m: h^{(n)}(m) \leq \tau$, and subsequently multiply $h^{\prime(n)}(m)$ by a constant such that $\sum h^{\prime(n)}(m)=\sum h^{(n)}(m)$.

4. Create $\left(\mathcal{H} \mu^{(n)}\right)(\mathbf{x})$ to fit new histogram $h^{\prime(n)}(m)$ by allocating pixels as per our original "top-down" ordering. The first $h^{\prime(n)}(M)$ voxel locations in our ordering take the highest available $\mu^{(n)}(\mathbf{x})=[M+0.5] w$. The next $h^{\prime(n)}(M$ $-1)$ voxel locations in our ordering will assume the gray value $\mu^{(n)}(\mathbf{x})=[M-0.5] w$, etc.

Step 3 effectively moves pixels from less- to morepopulated bins, encouraging peak formation.

The constant $\tau$ should be high enough to exclude noise from the histogram, but low enough to not eliminate any legitimate peaks. Low $\tau$ values correspond to relatively weak a priori information; if $w$ is sufficiently small then in the limiting case of $\tau=0,\left(\mathcal{H} \mu^{(n)}\right)(\mathbf{x}) \approx \mu^{(n)}(\mathbf{x})$ and our algorithm reduces to iterated $\mathrm{FBP}^{14}$ If the sample contains only a single, known material of constant density, then $\mathcal{H}$ may be replaced by a thresholding operator, and Eq. (3) becomes the binary tomography algorithm outlined in Ref. 10 .

The parameter $0<\gamma^{(n)} \leq 1$ is varied to ensure convergence as follows: (i) Each iteration is performed first with $\gamma^{(n)}=1$, and is then tested to ensure that the simulated projections $\left(\mathcal{P H} \mu^{(n)}\right)(\mathbf{r}, \theta)$ have not grown further away (in the L2 norm) from the measured projections $g(\mathbf{r}, \theta)$, when compared to the previous iteration. (ii) Should the test fail, the alterations made at this iteration are rejected, $\gamma_{n}$ is halved, and the iteration is attempted again. We iterate as per Eq. (3) until either no step is possible without reducing $\gamma$ past some predetermined limit, or the change in $\mu^{(n)}(\mathbf{x})$ falls below a predetermined accuracy threshold. When the reconstruction procedure terminates, $\left(\mathcal{H} \mu^{n}\right)(\mathbf{x})$ is returned as the solution.

Cone- and fan-beam imaging geometries are commonly used in lab-based CT setups. In principle, our method may be implemented in any imaging geometry for which we have knowledge of: (i) a projection operator $\mathcal{P}$, and (ii) a reconstruction operator $\mathcal{B}$ which is sufficiently well-behaved for lower numbers of projections (e.g., FBP, ${ }^{1}$ simultaneous iterative reconstruction technique, ${ }^{4}$ etc.)

We construct simulated "objects" by thresholding the well-known "Lena" image to form a one-, two-, or threematerial, structurally complex, $512 \times 512$ pixel phantom which is assumed to lie in the $x_{1}-x_{2}$ plane. We then simulate projections through this phantom, and perform a reconstruction using HB-CT (see Fig. 1). The number of bins in the histogram is determined based on the desired density resolution of the system. In every case in this letter, a histogram with $M=256$ bins was used, and $\tau$ was half the mean value 


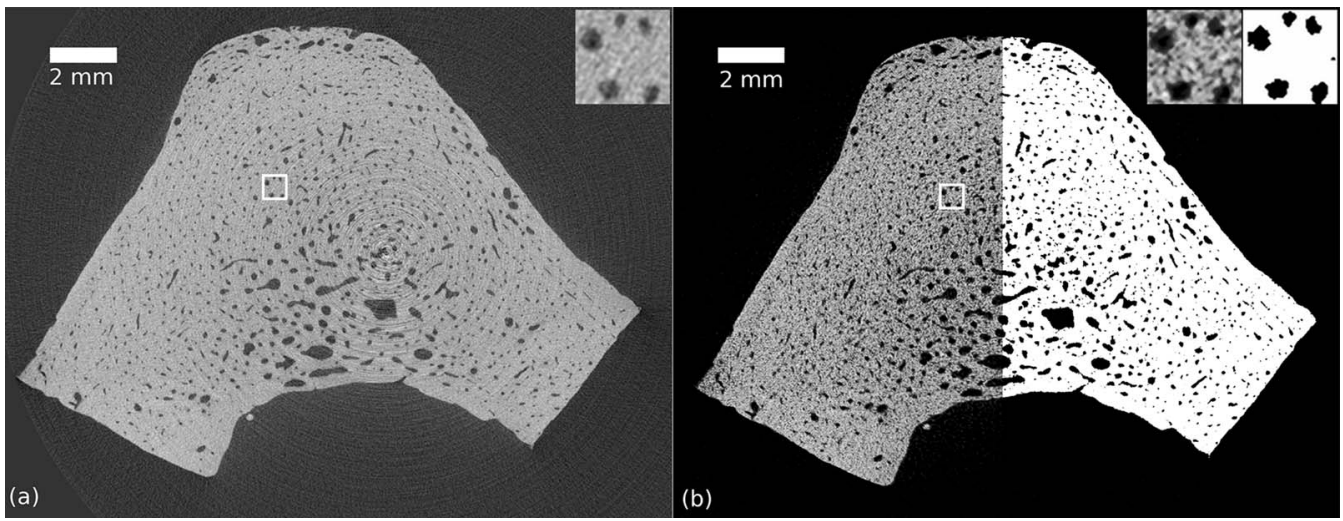

FIG. 2. A slice through reconstructions of cortical bone. Inset upper right of each image is magnified $4 \times$. (a) 3033 projections, total scanning time $\approx 2.75 \mathrm{~h}$, reconstructed via FBP; and (b) 300 projections, total scanning time $\approx 17 \mathrm{~min}$, reconstructed using HB-CT. The right half of this image has been thresholded, and a $3 \times 3$ median filter applied to remove single pixel noise. The thresholded and nonthresholded insets come from the same region within the sample.

of $h^{(n)}(m)$ (averaged across all bins). No effort was made to optimize $\tau$ for these particular cases.

We see that: (i) the minimum required number of projections for an accurate HB-CT reconstruction $\left(p_{\mathrm{h}}\right)$ increases with the complexity of the phantom; and (ii) when $p$ falls too low the behavior of the reconstruction algorithm varies unpredictably, thus establishing a lower limit on $p_{\mathrm{h}}$. It is likely that there are more sophisticated choices of $\mathcal{H}$ which will further improve the performance of HB-CT. If one can tolerate at least $\sim 4 \%$ of misclassified pixels, $p_{\mathrm{h}} \approx p_{\mathrm{ct}} / 10$, for one-, two-, or three-material objects with noise $<5 \%$.

We turn to an experimental implementation. The motivation: age-related bone loss leading to osteoporotic fracture, a major cause of mortality and morbidity in the elderly. ${ }^{15}$ The current deficit in understanding of age-related changes in 3D cortical bone microstructure is primarily due to methodological limitations. A specimen from the cortex of the femur of a 31 year old male was imaged at the SPring-8 BL20B2 synchrotron beamline, using monochromatic $37 \mathrm{keV} x$-rays, and with energy of and a detector with an effective pixel size of $11.8 \mu \mathrm{m}$. Ethical oversight of the collection process was by the Victorian Institute of Forensic Medicine, Human Research Ethics Committee (approval EC26/2000) and of the experimental procedure by the Human Research Ethics Committee of the Faculty of Medicine, Dentistry, and Health Science, at the University of Melbourne, Australia (HREC980139).

A $2000 \times 2000 \times 1200$ reconstruction was performed using $\operatorname{FBP}(p=3033)$, and 12 iterations of HB-CT (using only every tenth projection). Figure 2 shows excellent agreement between HB-CT and the "benchmark" FBP algorithm. Note that using every tenth projection decreases the effective signal-to-noise ratio by a factor of $\approx 3$. The strongly peaked histogram of the HB-CT reconstruction suggests a natural thresholding scheme. Accordingly, to give an idea of the microstructural information contained in Fig. 2, we have thresholded the right half of the HB-CT reconstruction, and applied a $3 \times 3$ median filter to the thresholded area to remove single pixel noise. Note that certain ring artifacts are more prominent in the FBP reconstruction. Because these artifacts comprise a relatively small number of voxels which assume unusually high or low values of $\mu(\mathbf{x})$, they are eliminated as noise by the HB-CT reconstruction procedure, given our choice of $\tau$. This demonstrates an additional beneficial feature of the proposed algorithm: by using the weak a priori information about the sample, it is capable of intrinsic denoising and segmentation of the reconstructed images. This test case demonstrates the following: (i) reconstruction in the presence of realistic density variation across the sample, (ii) reconstruction in the presence of realistic noise, and (iii) the application of HB-CT to a contemporary biological problem (osteoporosis research), and, more generally, to the rapid imaging of microstructure in porous materials.

G.R.M. acknowledges useful discussions with Dr. A. Kingston, Dr. T. Varslot, and Dr. A. Sheppard. G.R.M. acknowledges a Monash University School of Physics postgraduate publication award. C.D.L.T. acknowledges beam time at JASRI, and thanks Dr. K. Uesugi and Dr. N. Yagi. C.D.L.T. thanks Dr. C. Hall (MCSS) who was part of the team at SPring8 and a great source of advice, and the University of Melbourne for funding. D.M.P. acknowledges funding from the Australian Research Council.

${ }^{1}$ F. Natterer, The Mathematics of Computerized Tomography (Society for Industrial and Applied Mathematics, Philadelphia, 2001).

${ }^{2}$ D. M. Paganin, Coherent X-ray Optics (Oxford University Press, New York, 2006).

${ }^{3}$ Advances in Discrete Tomography and Its Applications, edited by G. T. Herman and A. Kuba (Birkhäuser, Boston, 2007).

${ }^{4}$ K. J. Batenburg, S. Bals, J. Sijbers, C. Kübel, P. A. Midgley, J. C. Hernandez, U. Kaiser, E. R. Encina, E. A. Coronado, and G. Van Tendeloo, Ultramicroscopy 109, 730 (2009).

${ }^{5}$ F. Natterer and F. Wübbeling, Mathematical Methods in Image Reconstruction (Society for Industrial and Applied Mathematics, Philadelphia, 2001).

${ }^{6}$ S. Weber, T. Schüle, J. Hornegger, and C. Schnörr, Lect. Notes Comput. Sci. 3322, 38 (2004).

${ }^{7}$ L. Hajdu and R. Tijdeman, Linear Algebr. Appl. 339, 147 (2001).

${ }^{8}$ A. Alpers, H. F. Poulsen, E. Knudsen, and G. T. Herman, J. Appl. Crystallogr. 39, 582 (2006).

${ }^{9}$ G. R. Myers, T. E. Gureyev, D. M. Paganin, and S. C. Mayo, Opt. Express 16, 10736 (2008)

${ }^{10}$ G. R. Myers, D. M. Paganin, T. E. Gureyev, and S. C. Mayo, Opt. Express 16, 908 (2008).

${ }^{11}$ P. Gritzmann, D. Prangenberg, S. de Vries, and M. Wiegelmann, Int. J. Imaging Syst. Technol. 9, 101 (1998).

${ }^{12}$ K. J. Batenburg and J. Sijbers, Pattern Recogn. 42, 2297 (2009).

${ }^{13}$ H. M. Goldman, C. D. L. Thomas, J. G. Clement, and T. G. Bromage, J. Anat. 206, 127 (2005).

${ }^{14}$ D. S. Lalush and B. M. W. Tsui, Med. Phys. 21, 1283 (1994).

${ }^{15}$ P. N. Sambrook, E. Seeman, S. R. Phillips, and P. R. Ebeling, Med. J. Aust. 176, S1 (2002) 